
\title{
Effects of Vitamin K in Periodontium
}

\author{
K. Malathi ${ }^{1}$, E. Amuthavalli ${ }^{2}$, R. Thanmanam ${ }^{3}$, V. Dasarathan ${ }^{4}$ \\ ${ }^{1,2,3,4}$ Department of Periodontics, Tamilnadu Government Dental College, Chennai, Tamilnadu, India
}

\begin{abstract}
Systemic diseases that affect generalized tissue throughout the body may also involve tissue within the periodontium. An important factor that modify the severity of a diseases is host resistance. Tissue resistance is modified by nutritional status and malnutrition is the most widespread immune suppressive and constitute a special risk group for severe periodontal pathogenesis . Synthesis of blood clotting factors in the liver is the only established function of vitamin $k$. This review discuss about vitamin $K$ and clinical implication of its deficiency in periodontal diseases.
\end{abstract}

Keywords: vitamin $\mathrm{K}$ - malnutrition -role in coagulation -periodontal disease

\section{Introduction}

Vitamin is an organic substance that occurs in food in small amounts and is necessary for several metabolic functions of the body. They act specially as coenzymes and precursors of co enzyme in the regulation of the smetabolic process. Henrickdam , a biochemist discovered this fat soluble blood clotting vitamin and named it Koagulation vitamin or vitamin K .K stands for Koagulation-the Danish word. Chemistry of Vitamin $\mathrm{K}$ represents it is a group of lipophilic and hydrophobic vitamin. There are two natural form of Vitamins, Vitamin K1,K2 .where K1 is from natural sources and $\mathrm{K} 2$ is from bacterial flora in the hepatic tissue. Vitamin $\mathrm{K} 3$ is chemically synthesized pro vitamin and is water soluable.

\section{Vitamin $\mathrm{K}$ as a growth factor}

As Vitamin $\mathrm{K}$ also acts as a growth factor for a Bifido bacterium strains, these bacteria may compete with P.gingivalis for Vitamin $\mathrm{K}$ in the oral cavity. Where bifidobacteia and P.gingivalis are cultured together, the concentration of Vitamin $\mathrm{K}$ comes down due to the utilization by /both bacteria[1]. Mycobacteria para tuberculosis was listed as Vitamin $\mathrm{K}$ requiring bacteria[2] .Bacteroides species are the most common bacteria that inhabit human intestinal tract. Strains such as bacteroidesfragilis synthesise Vitamin $\mathrm{K}$ and provides more significant amount of Vitamin $\mathrm{K}$ than E.coli strains[3]. Bacteria that inhabit the human oral cavity interact with each other, cooperating and competitively which correlates with periodontal health and disease .Lactic acid produced by streptococcus and actinomyces act as a carbon sources for Veillonella .Veillonella produces Vitamin K, this stimulates the growth of perio pathogens P.gingivalis[1]. .H2O2 produced by St.sanguis and other streptococcus inhibit the other bacterias including P.gingivalis. Vitamin $\mathrm{K}$ and its analogues has been accepted as growth factor for both P.gingivalis strains and Bifdidobacterium. Bifidobacterium inhibit P.gingivalis by reducing the concentration of its growth factor.

Dietary Sources of Vitamin K is found in green leafy vegetables such as kale and spinach, and appreciable amounts are also present in margarine and liver. Vitamin $\mathrm{K}$ is present in vegetable oils and is particularly rich in olive, canola, and soyabean oils[4]. Some amount is contributed by intestinal bacteria .Absorption takes place in small intestine in the presence of bile salts. The transportation from intestine is carried out through chylomicrons. Storage occurs in liver and from liver transportation to peripheral cells is carried out by binding with beta lipoproteins.

Fat malabsorption is associated with impaired absorption of vitamin $\mathrm{K}$ and other fat soluble vitamins. Vitamin $\mathrm{K}$ deficiency affect the coagulation process grossly as it results in bleeding and hemorrhages. Vitamin $\mathrm{K}$ absorption is also decreased by intake of mineral oil, bile acid sequestrants (Cholestyramine, Colestipol) and Orlistat (weight loss medication).Requirement increases in liver disorders, patients on prolonged antibiotic therapy, bile acidsequestrant (Cholestyramine, colestipol) and Orlistat (weight loss medication). The current daily average intake of vitamin $\mathrm{K}$ for adult women and men are $90 \mu \mathrm{g}$ and $120 \mu \mathrm{g}$ respectively.

The main functions of Vitamin $\mathrm{K}$ is Glutamate carboxylation in post synthetic modification of calcium binding proteins, by acting as a cofactor for an enzyme (Carboxylase).This carboxylase catalyzes the carboxylation of glutamic acid, and convert it in to gamma-carboxy glutamic acid (Gla). .It is important for the calcium-binding function of those Vitamin $\mathrm{K}$-dependent calcium binding proteins .Vitamin $\mathrm{k}$ dependent calcium binding proteins are clotting factor proteins and proteins found in bone, lung, kidney and also in placenta.

\section{Role of vitamin $K$ in coagulation}

The ability of Vitamin K-dependent clotting factors to bind calcium ions $\left(\mathrm{Ca}^{2+}\right)$ is needed for their activation in the coagulation cascade. Factors II (prothrombin), VII, IX, and $\mathrm{X}$ are vitamin $\mathrm{k}$ dependent clotting factor .Inactive form of these factors are synthesized in the liver and then they undergo post translational modifications, which imparts the negative charge, that promote calcium binding of these factors. Gamma-carboxyglutamate also chelates calcium ions, so it permits the binding of the blood clotting proteins to membranes [5]. 


\section{International Journal of Science and Research (IJSR)}

ISSN (Online): 2319-7064

Index Copernicus Value (2015): 78.96 | Impact Factor (2015): 6.391

\section{Vitamin K Cycle}

As it a fat-soluble vitamin, the body can store very little Vitamin $\mathrm{K}$ of it, and its stores are rapidly depleted without regular dietary intake. Because of its limited ability to store vitamin $\mathrm{K}$, the body recycles it through a process called the vitamin $\mathrm{K}$ cycle. Hence only a small amount of vitamin $\mathrm{K}$ is necessary to function in the gamma-carboxylation of proteins many times, decreasing the dietary requirement.. By the enzymatic reaction vitamin $\mathrm{K}$ hydroquinone (active form) is oxidized to the Epoxide. The initial form (hydroquinone form) is regenerated by two steps process. In First step, by an Epoxide reductase Vitamin $\mathrm{K}$ Epoxide is reduced to the quinone. By either the same reductase or by a vitamin $\mathrm{K}$ reductase(quinonereductase ) quinone is reduced to the active hydroquinone. Epoxide reductase requires reduced lipoamide for its action whereas the action of vitamin $\mathrm{K}$ reductase need NADPH[6].

\section{Vitamin K Antagonist}

Coagulation process is inhibited by Di cumarol and Warfarin through antagonism of the action of vitamin $\mathrm{K}$. Warfarin prevents the recycling of vitamin $\mathrm{K}$ and creates a functional vitamin $\mathrm{K}$ deficiency. Warfarin is a competitive inhibitor of Epoxide reductase. Hence vitamin $\mathrm{K}$ epoxides cannot be reduced, they accumulate and are excreted. This leads to formation of Abnormal precursor of prothrombin (pre prothrombin) which contain little or no carboxyglutamate, and is incapable of chelating calcium[7].The anticoagulant effect of vitamin $\mathrm{K}$ antagonists can be overcome by Large quantities of dietary or supplemental vitamin K . Patients taking anti coagulants drugs are cautioned not to consume a diet containing very large or highly variable quantities of vitamin $\mathrm{K}$. Warfarin therapy in pregnant woman can lead to Fetal Warfarin syndrome(fetal bone abnormalities)

\section{Synthesis of Bone Calcium-Binding Proteins}

The two bone proteins, osteocalcin and bonematrix gla protein containing gamma carboxy glutamate need vitamin $\mathrm{K}$ for their synthesis . Osteoblasts synthesis osteocalcin, which is regulated by the active form of vitamin D,. The vitamin Kdependent gamma-carboxylation of three glutamic acid residues is necessary for the mineral-binding capacity of osteocalcin ,following which osteocalcin tightly binds to calcium[8]. Hydroxyproline which is present in osteocalcinis dependent on both vitamins $\mathrm{K}$ and $\mathrm{C}$ for its synthesis; vitamin $\mathrm{D}$ also induces its synthesis .Matrix Gla protein- MGP found in bone, cartilage, and soft tissue, including blood vessels, facilitate normal bone growth and development and prevents the calcification of soft tissues and cartilages. Another anticoagulant protein synthesized by osteoblasts ,Protein S-is also vitamin K-dependent, but its role in bone metabolism is not clear. The inherited protein $\mathrm{S}$ deficiency in children causes problem related to increased blood clotting as well as decreased bone density. Glutamyl carboxylase, an enzyme that synthesis gamma-carboxyglutamate has vitamin $\mathrm{K}$ as its coenzyme .This gamma-carboxyglutamate determine whether calcium deposits in bones and blood vessels or not .

\section{Methods of assessment}

Vitamin K status can be assessed by:

The prothrombin time (PT) test measures the time required for blood to clot. A blood sample is mixed with citric acid and put in a fibrometer; delayed clot formation indicates a deficiency. This test is insensitive to mild deficiency, as the values do not change until the concentration of prothrombin in the blood has declined by at least $50 \%$..

Under carboxylated prothrombin(PIVKA-II )test; PT is unable to detect subclinical deficiencies that can be detected with PIVKA-II testing.

Urinary $\gamma$-carboxy glutamic acid levels: It responds to changes in dietary vitamin $\mathrm{K}$ intake. Several days are required before any change can be observed. Under carboxylated osteocalcin (ucoc) levels have been inversely correlated with stores of vitamin $\mathrm{K}$ and bone strength .

\section{Vitamin K deficiency}

Primary deficiency is rare in healthy adults because average diet does not lack vitamin $\mathrm{k}$. Newborn infants are at an increased risk of deficiency. Other populations with an increased prevalence of vitamin $\mathrm{K}$ deficiency include those who suffer from liver damage or disease (e.g. Alcoholics), cystic fibrosis, or inflammatory bowel diseases, or have recently had abdominal surgeries. Secondary vitamin K deficiency can occur in people with bulimia, those on stringent diets, and those taking anticoagulants. Other drugs such as salicylates, barbiturates, and cefamandole, also cause vitamin $\mathrm{k}$ deficiency although the mechanisms are still unknown. Vitamin K deficiency can result in coagulopathy, a bleeding disorder .Symptoms of $\mathrm{K}$ deficiency include anemia, bruising, nosebleeds and bleeding of the gums in both sexes and heavy menstrual bleeding in women. Osteoporosis and coronary heart disease are strongly associated with lower levels of $\mathrm{K}_{2}$ (menaquinone). Vitamin $\mathrm{K}_{2}$ intake level is inversely related to severe aortic calcification and all-cause mortality.A prolonged prothrombintime is usually the first laboratory test result to be abnormal inbleeding due to vitamin $\mathrm{K}$ deficiency due to reduction in Prothrombin, FVII, FIX, and FX levels. The diagnosis of bleeding due to vitamin $\mathrm{K}$ deficiency is confirmed if administration of vitamin $\mathrm{K}$ halts the bleeding and reduces the PT value.

\section{Vitamin $\mathbf{k}$-periodontal disease}

Vitamin K compounds have been found to be required for growth of Bacteroides melaninogenicus, an organism closely associated with periodontal diseases[3].It is speculated that a suitable antimetabolite of Vitamin $\mathrm{K}$ might interfere with the growth of this organism, and consequently, prevent the occurrence of periodontal disease .Vitamin $\mathrm{K}$ deficiency leads to coagulopathy because of inadequate synthesis of prothrombin and other clotting factors. The most common oral manifestation is gingival bleeding. Presence of inflammation and potential for subsequent attachment loss at 


\section{International Journal of Science and Research (IJSR) \\ ISSN (Online): 2319-7064}

Index Copernicus Value (2015): 78.96 | Impact Factor (2015): 6.391

bleeding sites occurs. After periodontal surgery and treated with antibiotics that kills intestinal bacteria leads to vitamin $\mathrm{k}$ deficiency with resulting blood loss and poor wound healing . In 1998 RAWLINSONet al performed cross sectional study investigating health and disease site from 18 patients with adult periodontitis aged 27-64 years .the study result reveals that levels of phylloquinone in GCF lower in health than disease sites .Since phylloquinone is an absolute growth requirement for black pigmented anaerobes many of which are implicated in periodontal disease. Vitamin $\mathrm{K}$ at disease site provide nutrional requirement favouring the growth of black pigmented anaerobes. In 2007 ,HOJO et al assessed the possibility that bifido bacteria compete with P. Gingivalis for their mutual growth factor vitamin $\mathrm{k}$. He concluded that bifidobacterium decreased vitamin $\mathrm{k}$ concentration and inhibit the growth of P.gingivalis by competing for the growth factor in the environment

\section{Management of periodontal patients with bleeding disorders;}

Pre-operative precautions include a detailed medical history, which include previous hemorrhagic episodes after trauma or surgery or even spontaneous bleeding a ,family history regarding hereditary bleeding disorders, history of current illnesses, such as hepatic and renal failure, and list of medications interfering with hemostasis, such as non steroidal anti-inflammatory drugs and antibiotics ,anticoagulation medications, such as coumarin ,heparin, aspirin, clopidogrel, and ticlodipine. Treatment protocol may have to be modified to minimize the risk of intra-operative and postoperative bleeding .Patients lacking vitamin $\mathrm{K}$, because of malabsorption syndrome, should receive vitamin $\mathrm{K}$ supplement before the dental appointment to restore liver function and the synthesis of coagulation factors. If the patient has liver failure, the dental management of the patient should involve platelet transfusion in a hospital setting. Anticoagulant medications reduce the risk of embolism and increase the probability of bleeding during and after the dental procedure. There are a number of case reports stating that serious thromboembolic episode in patients who discontinued taking the anticoagulant medications is three times higher than that of a bleeding event in patients who remained on the anticoagulant regimen.Pre-operative care of patients on anticoagulant therapy with coumarin involves the continuation, reduction or withdrawal of the medication. The decision should be based on the international normalized ratio value, the invasiveness and extent of dental procedure, current illnesses and medications. The international normalized ratio is a key component in the dental treatment of these patients. When the international normalized ratio is $\leq 3.5$, periodontal surgical procedures can be carried out on these patients in a dental office. When the international normalized ratio is $>3.5$, the anticoagulation regimen has to be adjusted. and should consult with the medical care provider and describe, in detail, the periodontal procedure and risk for bleeding. A safe approach entails reduction of the coumarin dose 2-3 days before the procedure and repetition of international normalized ratio testing the morning of the procedure to ensure that the value is $<4$.
International normalized ratio values can be normalized to 3.5 by making minor adjustments involving the reduction, but not discontinuation of coumarin.Entire withdrawal of coumarin is not recommended because of the rebound thrombotic effect noticed especially in patients with prosthetic cardiac valves when coumarinintakeis reinitiated. It takes up to 4 days for the international normalized ratio values to return to normal.Therefore, reducing the international normalized ratio value may increase the risk for thrombosis in patients with other concomitant illnesses such as liver and renal disease, and patients with alcohol consumption. Extensive-invasive periodontal surgical procedures in such patients should be performed in a hospital setting.Intravenous unfractionated heparin should be given as a substitute for coumarin .Unfractionated heparin can be interrupted 4-6 hours before the surgical procedure, thus substantially minimizing the time the patient is under a suboptimal level of anticoagulation and subsequently reducing the risk for a thromboembolic event .Anticoagulation treatment is resumed 12-18 hours after the dental procedure. Fractionated or low-molecular-weight heparin may provide an alternative substitute for coumarin, without the need for the patient to be admitted to hospital . Subcutaneous administration of low-molecular-weight heparin provides the benefit of conveniently adjusting the anticoagulation regimen at the point of care .

Intra operatively professional cleaning, and scaling and root planing, can be safely performed with the use of local anti fibrinolytic mouthwash, such as tranexamic acid or epsilon aminocaproic acid. Regional block anesthesia must be avoided. Local infiltration anesthesia with the use of vasoconstrictive agent is desirable.Lidocaine $2 \%$ with $1: 100,000$ epinephrine is adequate. Articaine 4\% with $1: 100,000$ epinephrine provides sufficientanesthesiafor surgery in the mandible.

Postoperative management is crucial for preventing bleeding. General recommendations emphasize the importance of good care of the surgical area. Rinsing is prohibited on the day of surgery and the healing site must be left undisturbed. Specific attention should be given to tongue movements interfering with healing and food intake. Liquids and a high-protein diet are strongly recommended. The use of anti fibrinolytic mouthwash is highly recommended the day after periodontal treatment. The regimen may comprise rinsing with $10 \mathrm{ml}$ of 4.8-5\%tranexamic acid solution, four times a day, for 2 minutes The rinsing can be carried out over a period of 2-5 days and may be extended up to 8 days[9] .Antibiotics, such as penicillin erythromycin, tetracycline, metronidazole, cephalosporins ,ampicillin and amoxicillin + clavulanic acid, potentiate the coumarin action. Acetaminophen can also interact with coumarin and its use must be limited to fewer than six tablets per week. Clindamycin should be the antibiotic of choice in these patients, and a lower dose of acetaminophen for a short period of time is the regimen recommended for postoperative pain control . When other medications are administered that affect coumarin bioavailability or metabolism, supplemental international normalized ratio tests are performed to evaluate the anticoagulation effect .The only use of vitamin $\mathrm{K}$ is, in prophylaxis and treatment of bleeding due to deficiency of

\section{Volume 6 Issue 12, December 2017}




\section{International Journal of Science and Research (IJSR) \\ ISSN (Online): 2319-7064}

Index Copernicus Value (2015): 78.96 | Impact Factor (2015): 6.391

clotting factors in the following situations. Dietary deficiency of vitamin $\mathrm{K}$ is very rare in adults .However, when occurs 5$10 \mathrm{mg} /$ day oral /parentral vitamin $\mathrm{K}$ rapidly correct the defects. In prolonged anti microbial therapy treatment should be in the same way as dietary deficiency .In obstructive jaundice and mal absorption syndromes; sprue, regional illeitis, vitamin k $10 \mathrm{mg}$ i.m./day or orally along with bile salts is essential. Liver diseases(viral hepatitis, cirrhosis) associated with bleeding responds poorly to vitamin $\mathrm{K}$ ,because of hepato cellular damage ,the synthesis of clotting factors is inadequate despite the presence of vitamin $\mathrm{K}$. However vitamin $\mathrm{K}$ may be of some use if the absorption had been affected due to lack of bile salt.

\section{Conclusion}

Vitamins are organic substances that are not synthesized by the body and are necessary for normal metabolism. Most of the fat soluble vitamins are absorbed in mid end distal ileum because of digestion of fat by bile and pancreatic lipase is required .Phylloquinone /vitamin K1 is one of the absolute growth requirement for obligate anaerobic microorganism which are believed to be among these major putative periodontal pathogens. Phylloquinone in the plant product present in normal sera in the order of $0.3-0.5 \mathrm{ng} / \mathrm{ml}$ .Nutritional deficiency and imbalance have the potential influence on the biological gradient and natural course of periodontal infection[10].Knowledge and physiology of vitamin $\mathrm{K}$ in human is far from complete. Diet or intestinal microflora in providing human requirement of vitamin $\mathrm{k}$ still remains to be un answered .In complex chemistry of the body without vitamin there is no life, with a deficiency of vitamin there is no health[11].

\section{References}

[1] Hojo1, S. Nagaoka1, S. Murata1, N. Taketomo1, T. Ohshima2 and N. Maeda .Reduction of vitamin K concentration by salivary bifido bacterium strains and their possible nutritional competition with Porphyromonas gingivalis K. Journal of Applied Microbiology ISSN; 1364-5072.

[2] Ronald Bentley and R. Meganathan .Biosynthesis of Vitamin K (Menaquinone ) in Bacteria. MICROBIOLOGICAL REVIEWS Sept. 1982; Vol. 46, No. 3; 241-280.

[3] Gibbons, R. J., and L. P. Engle. Vitamin K compounds in bacteria that are obligate anaerobes. Science1964; 146;1307-1309.

[4] Alison M. Daines, Richard J. Payne, Mark E. Humphries, and Andrew D. Abell .The Synthesis of Naturally Occurring Vitamin $\mathrm{K}$ and Vitamin K Analogues .Current Organic Chemistry2003;7;1-15.

[5] Presnell, s. R.; stafford, d. W. "the VITAMIN kDEPENDENT CARBOXYLASE".Journal of Thrombosis andhaemostasis jun2002;87 (6);937-946.

[6] Stafford, D. W.. "The vitamin K cycle". Journal of Thrombosis and Haemostasis .Aug 2005; 3 (8); 1873 187.
[7] LivHelgeland the action of vitamin $\mathrm{k}$ and coumarinanticoagulants . biochemical education1980; $8 ; 3$.

[8] Nishimoto, S K and Price. J BiolChem 1979; 254; $437-$ 441

[9] Philip Vassilopoulos \& Kent Palcanis. Bleeding disorders and periodontology. Periodontology 2000; Vol. 44; 2007;211-223.

[10]De paola\&Alfano; Triphasicnutrional analysis and dietary counselling.DCNA1976;20;622

[11] Enwonwo et al; cellular and molecular effect of malnutrition and their relevance to periodontal disease .J.clin,periodontal 1994;21;643-657.

\section{Author Profile}

Dr, Malathi. k. received the B..D.S . and M.D.S. degrees in periodontics from tamilnadu government dental college and hospital ,Chennai. Now she is working as H.O.D. and professor in the department of periodontics and tamil nadu government dental college and hospital Chennai. 